1 Hacettepe Journal of Mathematics and Statistics

holume 47 (1) (2018), 9-18

\title{
On the degree based topological indices of benzene ring embedded in P-type-surface in $2 \mathrm{D}$ network
}

\author{
Ali Ahmad*†
}

\begin{abstract}
The structure of any finite molecular graph which represent numerical quantities are known as topological indices. The importance of topological indices is generally linked with QSAR/QSPR. In this paper, we compute general Zagreb $\left(M_{\alpha}\right)$ index, general Randić connectivity $\left(R_{\alpha}\right)$ index, general sum-connectivity $\left(\chi_{\alpha}\right)$ index, atom-bond connectivity $(A B C)$ index and geometric-arithmetic $(G A)$ index, $A B C_{4}, G A_{5}$, multiple Zagreb indices and Zagreb polynomials indices of the benzene ring embedded in P-type-surface in 2D network.
\end{abstract}

Keywords: topological indices, multiple Zagreb indices, Zagreb polynomials, benzene ring.

2000 AMS Classification: 05C05, 05C12, 05C15, 05C31, 05C69.

Received: 09.12.2016 Accepted : 28.02.2017 Doi : 10.15672/HJMS.2017.443

\section{Introduction}

In this paper all graphs are finite, simple and undirected. Let $V(G)$ and $E(G)$ be the vertex set and edge set of a graph $G$. We follow the basic notation and terminology of graph theory as in [29]. The vertices $u, v \in V(G)$ are adjacent (or neighbors) if $u$ and $v$ are endpoints of $e \in E(G)$ and $e$ is incident with the vertices $u$ and $v$ and $e$ is said to connect $u$ and $v$. The set of all neighbors of a vertex $u$ of $G$ denoted by $N(u)$, is called the neighborhood of $v$. The degree of a vertex in an undirected simple graph is the number of edges incident with it. The degree of the vertex $u$ is denoted by $d_{u}$ and $S_{u}$ is the sum of degrees of all vertices adjacent to the vertex $u$. In other words $S_{u}=\sum_{v \in N(u)} d_{v}$, where $N(u)=\{v \in V(G): u v \in E(G)\}$.

The application of molecular structure descriptors is nowadays a standard procedure in the study of structure-property relations, especially in QSPR/QSAR study. In the latest couple of years, the amount of proposed nuclear descriptors is rapidly getting to

\footnotetext{
${ }^{*}$ College of Computer Science \& Information Systems, Jazan University, Jazan, KSA. Email: ahmadsms@gmail.com

†Corresponding Author.
} 
be a result of the creation significance of these descriptors. They interface the particular physico-substance properties of mixture blends. A most seasoned, most considered and most prominent topological record among all degree based topological lists is Randić index, which was presented by Randić in 1975 [28]. This record was discovered reasonable with the end goal of medication plan $[18,28]$. The numerical elements of Randić index incorporates its association with the standardized Laplacian framework $[5,6,16]$. The formal definition of Randić index of a graph $G$ is given as follows

$$
R(G)=\sum_{u v \in E(G)} \frac{1}{\sqrt{d_{u} d_{v}}} .
$$

Soon after the discovery of Randić index was introduced a general Randić index. It is denoted by $R_{\alpha}(G)$ and its formula is given as

$$
R_{\alpha}(G)=\sum_{u v \in E(G)}\left(d_{u} d_{v}\right)^{\alpha}
$$

where $\alpha$ is a nonzero real number. Zhou et al. [34] introduced the general sumconnectivity index $\chi_{\alpha}(G)$ and defined as

$$
\chi_{\alpha}(G)=\sum_{u v \in E(G)}\left(d_{u}+d_{v}\right)^{\alpha},
$$

where $\alpha$ is a real number. Shirdel et al. introduced a new degree based Zagreb index named as "hyper-Zagreb index" which is defined in [31], which is also known as general sum-connectivity index $\chi_{2}(G)$. The first general Zagreb index studied in [26].

$$
M_{\alpha}(G)=\sum_{u \in V(G)}\left(d_{u}\right)^{\alpha} .
$$

Estrada et al. invented atom-bond connectivity index which is abbreviated as $A B C$ index [13]. $A B C$ index is of much importance due to its correlation with the thermodynamic properties of alkanes, see $[12,19]$. The definition for $A B C$ index is as follows

$$
A B C(G)=\sum_{u v \in E(G)} \sqrt{\frac{d_{u}+d_{v}-2}{d_{u} d_{v}}} .
$$

The fourth version of $A B C$ index was introduced by Ghorbani and Hosseinzadeh [15] and defined as:

$$
A B C_{4}(G)=\sum_{u v \in E(G)} \sqrt{\frac{S_{u}+S_{v}-2}{S_{u} S_{v}}} .
$$

Another important degree based topological index is geometric-arithmetic index which is abbreviated as $G A$ index. It was invented by Vukičević and Furtula [33] and is of much importance due to its application to acyclic, unicyclic and bicyclic molecular graphs [10]. The formal definition of $G A$ index is as follows

$$
G A(G)=\sum_{u v \in E(G)} \frac{2 \sqrt{d_{u} d_{v}}}{d_{u}+d_{v}} .
$$

Recently the fifth version of $G A$ is introduced by Graovac el al. [20] and defined as:

$$
G A_{5}(G)=\sum_{u v \in E(G)} \frac{2 \sqrt{S_{u} S_{v}}}{S_{u}+S_{v}} .
$$


In [14], Ghorbani and Azimi defined first multiple Zagreb index $P M_{1}(G)$ and second multiple Zagreb index $P M_{2}(G)$ defined as:

$$
\begin{aligned}
& P M_{1}(G)=\prod_{u v \in E(G)}\left(d_{u}+d_{v}\right) . \\
& P M_{2}(G)=\prod_{u v \in E(G)}\left(d_{u} \times d_{v}\right) .
\end{aligned}
$$

These multiple Zagreb indices are studied for some chemical structures in $[4,11,17$, 25, 32], first Zagreb polynomial $M_{1}(G, x)$ and second Zagreb polynomial $M_{2}(G, x)$ are defined as:

$$
\begin{aligned}
& M_{1}(G, x)=\sum_{u v \in E(G)} x^{\left(d_{u}+d_{v}\right)} . \\
& M_{2}(G, x)=\sum_{u v \in E(G)} x^{\left(d_{u} \times d_{v}\right)} .
\end{aligned}
$$

We refer the interested reader for recent collection topological indices articles $[2,3,7,21$, $22,23,24,32]$.

\section{RESULTS AND DISCUSSION}

ÓKeeffe et al. [27] have distributed around a quarter century a letter managing two $3 \mathrm{D}$ systems of benzene one of the structure (Figure 1) was called 6.82P (additionally polybenzene) and has a place with the space gather $\operatorname{Im} 3 \mathrm{~m}$, comparing to the $P$-type surface. Actually this is inserting of the hexagon-fix in the surface of negative ebb and flow $P$. The P-type surface is coordinated to the Cartesian arranges in the Euclidean space. More about this intermittent surface the peruser can discover in $[8,9]$. This structure was required to be combined as $3 \mathrm{D}$ carbon solids: be that as it may, in our best learning, no such a combination was accounted for as such. This goal was to awaken the enthusiasm of researchers to the atomic acknowledgment of such pleasant thoughts in carbon nanoscience, as much as the graphenes were picked up a moment Nobel prize after $C_{60}$, also the immediate union of fullerenes is currently a reality see detail in $[1,32]$.

The graph $G$ of benzene ring embedded in the P-type-surface network is shown in Figure 1. The number of vertices in benzene ring embedded in the P-type-surface network are $24 m n$ among which $8 m n+4 m+4 n$ vertices are of degree 2 and $16 m n-4 m-4 n$ vertices are of degree 3 , by Table 1 .

Let $\delta(G)$ and $\Delta(G)$ be the minimum and maximum degree of $G$, respectively. The edge set $E(G)$ can be divided into several partitions: for any $i$ and $j, \delta(G) \leq i, j \leq \Delta(G)$, let $E_{i j}=\left\{e=u v \in E(G): d_{v}=i, d_{u}=j\right\}, e_{i, j}=\left|E_{i j}\right|$, and $V_{i}=\left\{v \in \bar{V}(G): d_{v}=i\right\}$, $n_{i}=\left|V_{i}\right|$.

Table 1. The vertex partition of graph $G$ based on degree of vertices

\begin{tabular}{lc}
\hline Degree of vertex & Number of vertices \\
\hline 2 & $8 m n+4 m+4 n$ \\
3 & $16 m n-4 m-4 n$ \\
\hline Total & $24 m n$ \\
\hline
\end{tabular}




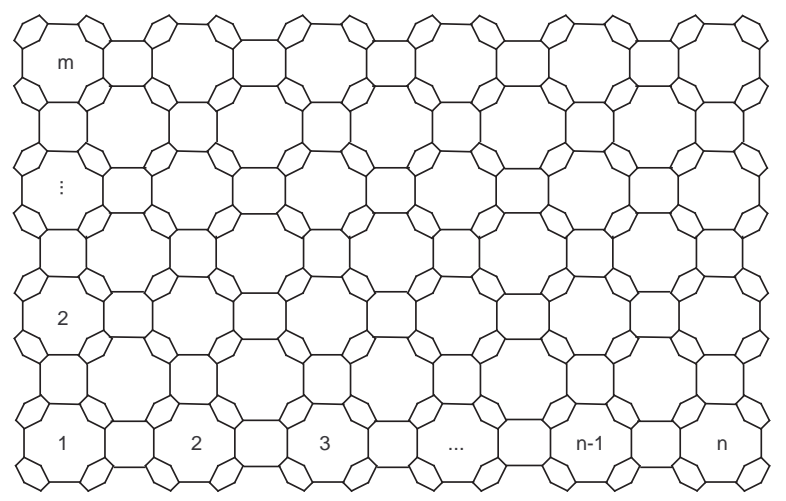

Figure 1. benzene ring embedded in the P-type-surface network.

Table 2. The edge partition of graph $G$ based on degree of end vertices of each edge.

\begin{tabular}{lc}
\hline$\left(d_{u}, d_{v}\right)$, where $u v \in E(G)$ & Number of edges \\
\hline$(2,2)$ & $4 m+4 n$ \\
$(2,3)$ & $16 m n$ \\
$(3,3)$ & $16 m n-6 m-6 n$ \\
\hline Total & $32 m n-2 m-2 n$ \\
\hline
\end{tabular}

\section{Main results}

3.1. Theorem. Let $G$ be a benzene ring embedded in the P-type-surface network, then

(1) $M_{\alpha}(G)=(8 m n+4 m+4 n) 2^{\alpha}+(16 m n-4 m-4 n) 3^{\alpha}$,

(2) $R_{\alpha}(G)=(4 m+4 n) 2^{2 \alpha}+(16 m n-6 m-6 n) 3^{2 \alpha}+(16 m n) 6^{\alpha}$,

(3) $\chi_{\alpha}(G)=(4 m+4 n) 2^{2 \alpha}+(16 m n) 5^{\alpha}+(16 m n-6 m-6 n) 6^{\alpha}$, where $\alpha$ is a real number.

Proof. The number of edges of benzene ring embedded in the P-type-surface network are $32 m n-2 m-2 n$. The edge partition based on the degree of the end vertices of each edge are shown in Table 2. Since, the formula of general Randić index is

$$
R_{\alpha}(G)=\sum_{u v \in E(G)}\left(d_{u} d_{v}\right)^{\alpha}
$$

this implies that $R_{\alpha}(G)=e_{2,2}(2 \times 2)^{\alpha}+e_{2,3}(2 \times 3)^{\alpha}+e_{3,3}(3 \times 3)^{\alpha}=(4 m+4 n) 4^{\alpha}+$ $(16 m n) 6^{\alpha}+(16 m n-6 m-6 n) 9^{\alpha}=(4 m+4 n) 2^{2 \alpha}+(16 m n-6 m-6 n) 3^{2 \alpha}+(16 m n) 6^{\alpha}$, and the formula of general sum-connectivity index is

$$
\chi_{\alpha}(G)=\sum_{u v \in E(G)}\left(d_{u}+d_{v}\right)^{\alpha}
$$

this implies that $\chi_{\alpha}(G)=e_{2,2}(2+2)^{\alpha}+e_{2,3}(2+3)^{\alpha}+e_{3,3}(3+3)^{\alpha}=(4 m+4 n) 2^{2 \alpha}+$ $(16 m n) 5^{\alpha}+(16 m n-6 m-6 n) 6^{\alpha}$. This completes the proof. 
3.2. Theorem. The atom-bound connectivity index $A B C$ of the benzene ring embedded in the P-type-surface network is given by

$$
A B C(G)=\left(8 \sqrt{2}+\frac{32}{3}\right) m n+(2 \sqrt{2}-4)(m+n) .
$$

Proof. The number of $e_{2,2}, e_{2,3}$ and $e_{3,3}$ edges are mentioned in Table 2 . Since, the atom-bond connectivity index is defined as

$$
A B C(G)=\sum_{u v \in E(G)} \sqrt{\frac{d_{u}+d_{v}-2}{d_{u} \times d_{v}}}
$$

this implies that

$$
A B C(G)=e_{2,2} \sqrt{\frac{2+2-2}{2 \times 2}}+e_{2,3} \sqrt{\frac{2+3-2}{2 \times 3}}+e_{3,3} \sqrt{\frac{3+3-2}{3 \times 3}} .
$$

By using the Table 2 , after simplification we get

$A B C(G)=\left(8 \sqrt{2}+\frac{32}{3}\right) m n+(2 \sqrt{2}-4)(m+n)$.

This completes the proof.

3.3. Theorem. The geometric-arithmetic index $G A$ of benzene ring embedded in the P-type-surface network is given by

$$
G A(G)=\left(16+\frac{32 \sqrt{6}}{5}\right) m n-2 m-2 n .
$$

Proof. The number of $e_{2,2}, e_{2,3}$ and $e_{3,3}$ edges are mentioned in Table 2. Since, the geometric-arithmetic index is defined as

$$
G A(G)=\sum_{u v \in E(G)} \frac{2 \sqrt{d_{u} \times d_{v}}}{d_{u}+d_{v}}
$$

this implies that $G A(G)=e_{2,2} \frac{2 \sqrt{2 \times 2}}{2+2}+e_{2,3} \frac{2 \sqrt{2 \times 3}}{2+3}+e_{3,3} \frac{2 \sqrt{3 \times 3}}{3+3}$.

By using the Table 2, we get

$G A(G)=(4 m+4 n) \frac{2 \times 2}{4}+(16 m n) \frac{2 \sqrt{6}}{5}+(16 m n-6 m-6 n) \frac{2 \times 3}{6}$.

After simplification we obtain

$G A(G)=\left(16+\frac{32 \sqrt{6}}{5}\right) m n-2 m-2 n$. This completes the proof.

In the next two theorems we calculated the fourth atom-bond connectivity index $A B C_{4}$ and the fifth geometric-arithmetic index $G A_{5}$. There are eight types of edges on degree based sum of neighbors vertices of each edge in the benzene ring embedded in the P-type-surface network. We use this partition of edges to calculate $A B C_{4}$ and $G A_{5}$ indices. Table 3 gives such types of edges of the benzene ring embedded in the P-typesurface network. The edge set $E(G)$ divided into eight edge partitions based on degree of end vertices.

The first edge partition $E_{4,5}(G)$ contains 8 edges $u v$, where $S_{u}=4, S_{v}=5$ and $m_{4,5}=\left|E_{4,5}(G)\right|$. The second edge partition $E_{5,5}(G)$ contains $4 m+4 n-8$ edges $u v$, where $S_{u}=S_{v}=5$ and $m_{5,5}=\left|E_{5,5}(G)\right|$. The third edge partition $E_{5,7}(G)$ contains $4 m+4 n$ edges $u v$, where $S_{u}=5, S_{v}=7$ and $m_{5,7}=\left|E_{5,7}(G)\right|$. The fourth edge partition $E_{5,8}(G)$ contains $4 m+4 n-8$ edges $u v$, where $S_{u}=5, S_{v}=8$ and $m_{5,8}=\left|E_{5,8}(G)\right|$. The fifth edge partition $E_{6,7}(G)$ contains $4 m+4 n$ edges $u v$, where $S_{u}=6, S_{v}=7$ and $m_{6,7}=\left|E_{6,7}(G)\right|$. The sixth edge partition $E_{6,8}(G)$ contains $16 m n-12 m-12 n+8$ edges $u v$, where $S_{u}=6, S_{v}=8$ and $m_{6,8}=\left|E_{6,8}(G)\right|$. The seventh edge partition $E_{7,7}(G)$ contains $2 m+2 n$ edges $u v$, where $S_{u}=S_{v}=7$ and $m_{7,7}=\left|E_{7,7}(G)\right|$. The eighth edge partition $E_{8,8}(G)$ contains $16 m n-8 m-8 n$ edges $u v$, where $S_{u}=S_{v}=8$ and $m_{8,8}=\left|E_{8,8}(G)\right|$. 
Table 3. The edge partition of graph $G$ based on degree sum of neighbor vertices of end vertices of each edge.

\begin{tabular}{lc}
\hline$\left(S_{u}, S_{v}\right)$, where $u v \in E(G)$ & Number of edges \\
\hline$(4,5)$ & 8 \\
$(5,5)$ & $4 m+4 n-8$ \\
$(5,7)$ & $4 m+4 n$ \\
$(5,8)$ & $4 m+4 n-8$ \\
$(6,7)$ & $4 m+4 n$ \\
$(6,8)$ & $16 m n-12 m-12 n+8$ \\
$(7,7)$ & $2 m+2 n$ \\
$(8,8)$ & $16 m n-8 m-8 n$ \\
\hline Total & $32 m n-2 m-2 n$ \\
\hline
\end{tabular}

3.4. Theorem. The fourth atom-bound connectivity index $A B C_{4}$ of the benzene ring embedded in the P-type-surface network is given by $A B C_{4}(G)=2(4+\sqrt{14}) m n+\left(\frac{\sqrt{110}}{5}-\right.$ $\left.\frac{3 \sqrt{14}}{7}-6+\frac{2 \sqrt{462}}{21}+\frac{4 \sqrt{3}}{7}+\frac{8 \sqrt{2}}{5}\right)(m+n)+\frac{4 \sqrt{35}}{5}+4-\frac{16 \sqrt{2}}{5}-\frac{2 \sqrt{110}}{5}$.

Proof. Let $m_{i, j}$ denotes the number of edges of the benzene ring embedded in the P-typesurface network with $i=S_{u}$ and $j=S_{v}$. It is easy to see that the summation of degree of edge endpoints of given graph has eight edge types $m_{4,5}, m_{5,5}, m_{5,7}, m_{5,8}, m_{6,7}, m_{6,8}$, $m_{7,7}$ and $m_{8,8}$ that are shown in Table 3. The fourth atom-bound connectivity index $A B C_{4}$ is defined as:

$$
A B C_{4}(G)=\sum_{u v \in E(G)} \sqrt{\frac{S_{u}+S_{v}-2}{S_{u} \times S_{v}}}
$$

this implies that

$$
\begin{aligned}
& A B C_{4}(G)=m_{4,5} \sqrt{\frac{4+5-2}{4 \times 5}}+m_{5,5} \sqrt{\frac{5+5-2}{5 \times 5}}+m_{5,7} \sqrt{\frac{5+7-2}{5 \times 7}}+m_{5,8} \sqrt{\frac{5+8-2}{5 \times 8}}+m_{6,7} \sqrt{\frac{6+7-2}{6 \times 7}}+ \\
& m_{6,8} \sqrt{\frac{6+8-2}{6 \times 8}}+m_{7,7} \sqrt{\frac{7+7-2}{7 \times 7}}+m_{8,8} \sqrt{\frac{8+8-2}{8 \times 8}} .
\end{aligned}
$$

By using the Table 3 , we get

$$
\begin{gathered}
A B C_{4}(G)=8 \sqrt{\frac{7}{20}}+(4 m+4 n-8) \sqrt{\frac{8}{25}}+(4 m+4 n) \sqrt{\frac{10}{35}}+(4 m+4 n-8) \sqrt{\frac{11}{40}}+ \\
(4 m+4 n) \sqrt{\frac{11}{42}}+(16 m n-12 m-12 n+8) \sqrt{\frac{12}{48}}+(2 m+2 n) \sqrt{\frac{12}{49}}+(16 m n-8 m-8 n) \sqrt{\frac{14}{64}} .
\end{gathered}
$$

After simplification, we obtain

$A B C_{4}(G)=2(4+\sqrt{14}) m n+\left(\frac{\sqrt{110}}{5}-\frac{3 \sqrt{14}}{7}-6+\frac{2 \sqrt{462}}{21}+\frac{4 \sqrt{3}}{7}+\frac{8 \sqrt{2}}{5}\right)(m+n)+\frac{4 \sqrt{35}}{5}+$ $4-\frac{16 \sqrt{2}}{5}-\frac{2 \sqrt{110}}{5}$.

Which completes the proof.

3.5. Theorem. The fifth geometric-arithmetic index $G A_{5}$ of the benzene ring embedded in the P-type-surface network is given by

$G A_{5}=16\left(1+\frac{4 \sqrt{3}}{7}\right) m n+\left(-2+\frac{2}{3} \sqrt{35}+\frac{16}{13} \sqrt{10}+\frac{8}{13} \sqrt{42}-\frac{48}{7} \sqrt{3}\right)(m+n)-8+\frac{32 \sqrt{3}}{7}+$ $\frac{16 \sqrt{20}}{9}-\frac{32 \sqrt{10}}{13}$.

Proof. Let $m_{i, j}$ denotes the number of edges of the benzene ring embedded in the P-typesurface network with $i=S_{u}$ and $j=S_{v}$. It is easy to see that the summation of degree of edge endpoints of given graph has eight edge types $m_{4,5}, m_{5,5}, m_{5,7}, m_{5,8}, m_{6,7}, m_{6,8}$, $m_{7,7}$ and $m_{8,8}$ that are shown in Table 3. The fifth geometric-arithmetic index $G A_{5}$ is 
defined as:

$$
G A_{5}(G)=\sum_{u v \in E(G)} \frac{2 \sqrt{S_{u} \times S_{v}}}{S_{u}+S_{v}} .
$$

This implies that $G A_{5}(G)=m_{4,5} \frac{2 \sqrt{4 \times 5}}{4+5}+m_{5,5} \frac{2 \sqrt{5 \times 5}}{5+5}+m_{5,7} \frac{2 \sqrt{5 \times 7}}{5+7}+m_{5,8} \frac{2 \sqrt{5 \times 8}}{5+8}+$ $m_{6,7} \frac{2 \sqrt{6 \times 7}}{6+7}+m_{6,8} \frac{2 \sqrt{6 \times 8}}{6+8}+m_{7,7} \frac{2 \sqrt{7 \times 7}}{7+7}+m_{8,8} \frac{2 \sqrt{8 \times 8}}{8+8}$. By using the Table 3, we get

$G A_{5}(G)=8 \frac{2 \sqrt{20}}{9}+(4 m+4 n-8) \frac{2 \sqrt{25}}{10}+(4 m+4 n) \frac{2 \sqrt{35}}{12}+(4 m+4 n-8) \frac{2 \sqrt{40}}{13}+(4 m+$ $4 n) \frac{2 \sqrt{42}}{13}+(16 m n-12 m-12 n+8) \frac{2 \sqrt{48}}{14}+(2 m+2 n) \frac{2 \sqrt{49}}{14}+(16 m n-8 m-8 n) \frac{2 \sqrt{64}}{16}$.

After simplification we obtain

$G A_{5}(G)=16\left(1+\frac{4 \sqrt{3}}{7}\right) m n+\left(-2+\frac{2}{3} \sqrt{35}+\frac{16}{13} \sqrt{10}+\frac{8}{13} \sqrt{42}-\frac{48}{7} \sqrt{3}\right)(m+n)-8+$ $\frac{32 \sqrt{3}}{7}+\frac{16 \sqrt{20}}{9}-\frac{32 \sqrt{10}}{13}$. Which completes the proof.

We compute hyper-Zagreb index $H M(G)$, first multiple Zagreb index $P M_{1}(G)$, second multiple Zagreb index $P M_{2}(G)$, Zagreb polynomials $M_{1}(G, x), M_{2}(G, x)$ for benzene ring embedded in the P-type-surface network in the following theorem.

3.6. Theorem. Let $G$ be a benzene ring embedded in the P-type-surface network, then

(1) $H M(G)=976 m n-152 m-152 n$,

(2) $P M_{1}(G)=2^{16 m n+2 m+2 n} \times 3^{16 m n-6 m-6 n} \times 5^{16 m n}$

(3) $P M_{2}(G)=2^{16 m n+8 m+8 n} \times 3^{48 m n-12 m-12 n}$,

(4) $M_{1}(G, x)=4(m+n) x^{4}+16 m n x^{5}+(16 m n-6 m-6 n) x^{6}$,

(5) $M_{2}(G, x)=4(m+n) x^{4}+16 m n x^{6}+(16 m n-6 m-6 n) x^{9}$.

Proof. Let $G$ be a benzene ring embedded in the P-type-surface network. The edge set $E(G)$ divided into three edge partitions based on degree of end vertices. The first edge partition $E_{1}(G)$ contains $4 m+4 n$ edges $u v$, where $d_{u}=d_{v}=2$. The second edge partition $E_{2}(G)$ contains $16 m n$ edges $u v$, where $d_{u}=2, d_{v}=3$. The third edge partition $E_{3}(G)$ contains $16 m n-6 m-6 n$ edges $u v$, where $d_{u}=d_{v}=3$. Since,

$$
\begin{aligned}
H M(G) & =\sum_{u v \in E(G)}\left(d_{u}+d_{v}\right)^{2} \\
& =\sum_{u v \in E_{1}(G)}\left[d_{u}+d_{v}\right]^{2}+\sum_{u v \in E_{2}(G)}\left[d_{u}+d_{v}\right]^{2}+\sum_{u v \in E_{3}(G)}\left[d_{u}+d_{v}\right]^{2} \\
& =e_{2,2}(2+2)^{2}+e_{2,3}(2+3)^{2}+e_{3,3}(3+3)^{2} .
\end{aligned}
$$

After putting the values of edge partitions, we get

$H M(G)=976 m n-152 m-152 n$.

Since,

$$
\begin{aligned}
P M_{1}(G) & =\prod_{u v \in E(G)}\left(d_{u}+d_{v}\right) \\
& =\prod_{u v \in E_{1}(G)}\left(d_{u}+d_{v}\right) \times \prod_{u v \in E_{2}(G)}\left(d_{u}+d_{v}\right) \times \prod_{u v \in E_{3}(G)}\left(d_{u}+d_{v}\right) \\
& =(2+2)^{\left|E_{1}(G)\right|} \times(2+3)^{\left|E_{2}(G)\right|} \times(3+3)^{\left|E_{3}(G)\right|} \\
& =(4)^{4 m+4 n} \times(5)^{16 m n} \times(6)^{16 m n-5 m-6 n} .
\end{aligned}
$$


Now, since

$$
\begin{aligned}
P M_{2}(G) & =\prod_{u v \in E(G)}\left(d_{u} \times d_{v}\right) \\
& =\prod_{u v \in E_{1}(G)}\left(d_{u} \times d_{v}\right) \times \prod_{u v \in E_{2}(G)}\left(d_{u} \times d_{v}\right) \times \prod_{u v \in E_{3}(G)}\left(d_{u} \times d_{v}\right) \\
& =(4)^{\left|E_{1}(G)\right|} \times(6)^{\left|E_{2}(G)\right|} \times(9)^{\left|E_{3}(G)\right|} \\
& =(4)^{4 m+4 n} \times(6)^{16 m n} \times(9)^{16 m n-6 m-6 n} .
\end{aligned}
$$

After simplification we get

$$
\begin{aligned}
P M_{2}(G)=2^{16 m n+8 m+8 n} \times 3^{48 m n-12 m-12 n} \text {. As, } & x_{1}^{\left(d_{u}+d_{v}\right)} \\
& =\sum_{u v \in E(G)} x^{\left(d_{u}+d_{v}\right)}+\sum_{u v \in E_{2}(G)} x^{\left(d_{u}+d_{v}\right)}+\sum_{u v \in E_{3}(G)} x^{\left(d_{u}+d_{v}\right)} \\
& =\sum_{u v \in E_{1}(G)} x^{2+2}+\sum_{u v \in E_{2}(G)} x^{2+3}+\sum_{u v \in E_{3}(G)} x^{3+3} \\
& =\left|E_{1}(G)\right| x^{4}+\left|E_{2}(G)\right| x^{5}+\left|E_{3}(G)\right| x^{6} \\
& =(4 m+4 n) x^{4}+16 m n x^{5}+(16 m n-6 m-6 n) x^{6} .
\end{aligned}
$$

As

$$
\begin{aligned}
M_{2}(G, x) & =\sum_{u v \in E(G)} x^{\left(d_{u} \times d_{v}\right)} \\
& =\sum_{u v \in E_{1}(G)} x^{\left(d_{u} \times d_{v}\right)}+\sum_{u v \in E_{2}(G)} x^{\left(d_{u} \times d_{v}\right)}+\sum_{u v \in E_{3}(G)} x^{\left(d_{u} \times d_{v}\right)} \\
& =\sum_{u v \in E_{1}(G)} x^{4}+\sum_{u v \in E_{2}(G)} x^{6}+\sum_{u v \in E_{3}(G)} x^{9}
\end{aligned}
$$

By putting the values, we obtain

$M_{2}(G, x)=4(m+n) x^{4}+16 m n x^{6}+(16 m n-6 m-6 n) x^{9}$.

Which completes the proof.

\section{Closing Remarks}

In this paper, we deal with benzene ring embedded in the P-type-surface network and studies their topological indices. We determined first general Zagreb index $M_{\alpha}$, general Randić connectivity index $R_{\alpha}$, general sum-connectivity index $\chi_{\alpha}$, atom-bond connectivity index $A B C$, geometric-arithmetic index $G A$, fourth atom-bond connectivity index $A B C_{4}$, fifth geometric-arithmetic index $G A_{5}$, hyper-Zagreb index $H M(G)$, first multiple Zagreb index $P M_{1}(G)$, second multiple Zagreb index $P M_{2}(G)$ and Zagreb polynomials $M_{1}(G, x), M_{1}(G, x)$.

In future, we are intrigued to design some incipient architectures/networks and then study their topological indices which will be quite auxiliary to understand their underlying topological.

\section{References}

[1] K. Yu. Amsharov, M. Jansen, A $C_{78}$ Fullerene Precursor: Toward the Direct Synthesis of Higher Fullerenes, J. Org. Chem., 73, 2931-2934, 2008. 
[2] M. Bača, J. Horvràthovà, M. Mokrišovà, A. Suhànyiovà, On topological indices of fullerenes, Appl. Math. Comput., 251, 154-161, 2015.

[3] A. Q. Baig, M. Imran, H. Ali, S. U. Rehman, Computing topological polynomial of certain nanostructures, J. Optoelectron. Adv. Mat., 17(5-6), 877-883, 2015.

[4] B. Borovicanin, On the extremal Zagreb indices of trees with given number of segments or given number of branching vertices, MATCH Commun. Math. Comput. Chem., 74(1), 57-79, 2015.

[5] Ş. B. Bozkurt, A. D. Güngör, I. Gutman, and A. S. Çevik, Randić matrix and Randić energy, MATCH Commun. Math. Comput. Chem., 64, 239-250, 2010.

[6] M. Cavers, S. Fallat, and S. Kirkland, On the normalized Laplacian energy and general Randić index R-1 of graphs, Lin. Algebra Appl., 433, 172-190, 2010.

[7] A.A. Dobrynin, R. Entringer, I. Gutman, Wiener index of trees: theory and applications, Acta Appl. Math., 66, 21-249, 2001.

[8] M. V. Diudea (Ed.), Nanostructures, Novel Architecture, NOVA, New York, 2005.

[9] M. V. Diudea, Cs. L. Nagy, Periodic Nanostructures, Springer, Dordrecht, 2007.

[10] Z. Du, B. Zhou, and N. Trinajstić, On Geometric-Arithmetic Indices of (Molecular) Trees, Unicyclic Graphs and Bicyclic Graphs, MATCH Commun. Math. Comput. Chem., 66, 681697, 2011.

[11] M. Eliasi, A. Iranmanesh, I. Gutman, Multiplicative version of first Zagreb index, MATCH Commun. Math. Comput. Chem., 68, 217-230, 2012.

[12] E. Estrada, Atombond connectivity and the energetic of branched alkanes, Chem. Phys. Lett., 463, 422-425, 2008.

[13] E. Estrada, L. Torres, L. Rodriguez, I. Gutman, An atom-bond connectivity index: modelling the enthalpy of formation of alkanes, Indian J. Chem., 37A, 849-855, 1998.

[14] M. Ghorbani, N.Azimi, Note on multiple Zagreb indices, Iran. J. Math. Chem., 3(2), 137$143,2012$.

[15] M. Ghorbani, M. A. Hosseinzadeh, Computing $A B C_{4}$ index of nanostar dendrimers, Optoelectron. Adv. Mater. Rapid Commun., 4(9), 1419-1422, 2010.

[16] I. Gutman, B. Furtula, and Ş. B. Bozkurt, On Randić energy, Lin. Algebra Appl., 442, 50-57, 2014.

[17] I. Gutman, B. Furtula, Z. K. Vukicevic, G. Popivoda, On Zagreb Indices and Coindices, MATCHCommun. Math. Comput. Chem., 74(1), 5-16, 2015.

[18] I. Gutman, B. Ruščić, N. Trinajstić, and C. F. Wilcox, Graph Theory and Molecular Orbitals. XII. Acyclic Polyenes, J. Chem. Phys., 62(9), 3399-3405, 1975.

[19] I. Gutman, J. Tošović, S. Radenković, and S. Marković, On atom-bond connectivity index. and its chemical applicability, Indian J. Chem., 51A, 690-694, 2012.

[20] A. Gravovac, M. Ghorbani, M. A. Hosseinzadeh, Computing fifth geometric-arithmatic index $\mathrm{ABC}_{4}$ index of nanostar dendrimers, Optoelectron. Adv. Mater. Rapid Commun., 4(9), 1419-1422, 2010.

[21] S. Hayat, M. Imran, On some degree based topological indices of certain nanotubes, J. Comput. Theor. Nanosci., 12(8), 1599-1605, 2015.

[22] S. Hayat, M. Imran, Computation of topological indices of certain networks, Appl. Math. Comput., 240, 213-228, 2014.

[23] S. Hayat, M. Imran, Computation of certain topological indices of nanotubes, J. Comput. Theor. Nanosci., 12(1), 70-76, 2015.

[24] S. Hayat, M. Imran, Computation of certain topological indices of nanotubes covered by $C_{5}$ and $C_{7}$, Comput. Theor. Nanosci., 12(4), 533-541, 2015.

[25] X. Li, Y. Shi, A survey on the Randic index, MATCHCommun. Math. Comput. Chem., 59(1), 127-156, 2008.

[26] X. Li, H. Zhao, Trees with the first three smallest and largest generalized topological indices, MATCH Commun. Math. Comput. Chem., 50, 57-62, 2004.

[27] M. ÓKeeffe, G. B. Adams, O. F. Sankey, Predicted new low energy forms of carbon, Phys. Rev. Lett., 68, 2325-2328, 1992.

[28] M. Randić, On characterization of molecular branching., J. Am. Chem. Soc., 97, 6609-6615, 1975. 
[29] K. H. Rosen, Discrete Mathematics and its applications, The McGraw-Hill Companies, Inc., seventh edition, 2012.

[30] L. T. Scott, Methods for the chemical synthesis of fullerenes, Angew. Chem. Int. Ed., 43, 4994-5007, 2004.

[31] G. H. Shirdel, H. R. Pour, A. M. Sayadi, The Hyper-Zagreb index of graph operations, Iran. J. Math. Chem., 4(2), 213-220, 2013.

[32] Y. Shi, Note on two generalizations of the Randić index, Appl.Math.Comput., 265, 1019$1025,2015$.

[33] D. Vukičević and B. Furtula, J. Math. Chem. 46, 1369-1376, 2009.

[34] B. Zhou, N. Trinajstic, On general sum-connectivity index, J. Math. Chem., 47, 210-218, 2010. 